\title{
ESTUDIO DESCRIPTIVO DE LOS TUMORES TESTICULARES GERMINALES: 13 AÑOS DE EXPERIENCIA EN EL ÁREA DE SALUD DE BADAJOZ.
}

Fátima Lallave Martín', María Lomas Garrido², Eduardo Laguna Alvarez', Simón Asuar Aydillo', Jesús Murillo Mirat', Antonio Ramírez Zambrana? y Juan Luis Molina Suarez?.

Servicio de Urología' y Servicio de Oncología Médica². Hospital Infanta Cristina. Complejo Hospitalario Universitario de Badajoz. España.

Resumen.- OBJETIVO: Descripción de la incidencia, las características tumorales y los resultados de la terapéutica aplicada en los tumores germinales testiculares diagnosticados en nuestro Centro.

MÉTODO: Estudio retrospectivo de los 66 casos de tumores germinales diagnosticados en el Área de Salud de Badajoz en el período comprendido entre 1993 y 2005.

RESULTADOS: La edad media del diagnóstico de los tumores germinales de testículo (TGT) fue de 32 años

Fátima Lallave Martín

Av. Puente Real, 2 Bajo E

06007 Badajoz. (España).

falma75@terra.es

Trabajo recibido: 30 de diciembre 2006 (rango 16-80 años), presentando una edad más precoz los pacientes con tumores no seminomatosos (TGNS), media de 30 años. En un $86.5 \%$ de los paciente no se encontraron factores de riesgo relacionados con el diagnóstico de TGT. El hallazgo de una masa escrotal fue el síntoma mas frecuente y el tumor se localizó en mayor proporción en el testículo izquierdo (51.5\%). Los tumores germinales no seminomatosos fueron la histopatología más común (64.8\%). En el grupo de los tumores seminomatosos (TGTS), el estadio I (72\%) fué el más diagnosticado frente al $68.5 \%$ de los no seminomatosos. Los estadios II-III se dieron en el $34.4 \%$ de los TGNS y $28 \%$ de los seminomas, conllevando peor pronóstico. El 92\% de los pacientes recibió tratamiento adyuvante con quimioterapia y/o radioterapia, y se realizó cirugía curativa como único tratamiento en el $8 \%$ restante de los pacientes. Se practicó cirugía de masas residuales en 5 pacientes (estadios IIb, Ilc y IIIa). Ocho de los 66 casos se han perdido en el seguimiento. De los 58 pacientes restantes, donde fue posible el control de la evolución, 53 pacientes están libre de enfermedad, 18 de ellos con más de 5 años de seguimiento.

CONCLUSIONES: En los últimos años se verifica un aumento de la incidencia de TGT, sobre todo a expensa de los TGNS. Sin embargo, ha disminuido el diagnóstico en fase avanzada de la enfermedad en favor de estadios iniciales que confieren un mejor pronóstico para el paciente. Los protocolos de tratamiento oncológicos utilizados proporcionan una alta tasa de curabilidad, aunque debido a la historia natural de tumor, es necesario un seguimiento a largo plazo.

Palabras clave: Tumor germinal. Incidencia. Cáncer testicular. 
Summary.- OBJECTIVES: To describe the incidence of germ cell testicular tumors in our Center, their characteristics and therapy results.

METHODS: Retrospective study of 66 cases of germ cell testicular tumors diagnosed in the Health Area of Badajoz between 1993 and 2005.

RESULTS: Mean age of the time of diagnosis was 32 years (range 16-80 years), presenting a younger age patients with non seminomatous germ cell tumors (NSGCT) (mean age 30 years). $86.5 \%$ of the patients did not have risk factors associated with the diagnosis of germ cell testicular tumor. Testicular mass was the most frequent symptom, and a higher proportion of tumors were located in the left testicle (51.5\%). Non seminomatous germ cell tumors were the most frequent histological type (64.8\%). Stage I (72\%) was the most frequent stage in the group of seminomatous tumors, in comparison with $68.5 \%$ of non seminomatous tumors. Stages II-III appeared in $34.4 \%$ of the NSGCT and $28 \%$ of seminomatous, having worse prognosis.

92\% of the patients received adjuvant treatment with chemotherapy and/or radiotherapy, and curative surgery was the only treatment in the remainder $8 \%$. Residual mass surgery was undertaken in five patients (stages Ila, Ilc and IIla). Eight of the 66 cases were lost for follow-up. Fifty-three of the 58 patients with follow-up are disease-free, 18 of them with more than five years of follow-up.

CONCLUSIONS: An increased incidence of germ cell testicular tumors have been verified over last years, mainly NSGCT. Nevertheless, the diagnosis of advanced stages of the disease has diminished in favour of initial stages, which have a better prognosis for the patient. Oncologycal treatment protocols have high cure rates, although a long-term follow-up is needed due to the natural history of these tumors.

Keywords: Germ cell tumor. Incidence. Testicular cancer.

\section{INTRODUCCIÓN}

Las neoplasias testiculares suponen entre el $1-3 \%$ de los tumores del varón, aunque la incidencia se ha duplicado en los últimos 40 años. Son los tumores mas frecuentes en los varones jóvenes (20-40 años), por tanto inciden en la etapa mas productiva del individuo, con altos costes económicos, sociales y emocionales.
Se derivan en su mayoría de las células germinales testiculares (TGT) y sólo un 5\% corresponderían a tumores dependiente del estroma testicular y metastásicos $(1,2)$.

Estas neoplasias son un modelo de malignidad curable; incluso en los casos de enfermedad metastásica. Las tasas de curaciones superan el $80 \%$, considerando todos los estadios.

El objetivo del trabajo es analizar las características de nuestros pacientes, así como los resultados de los tratamientos aplicados y supervivencia, durante los últimos 13 años.

\section{MATERIAL Y MÉTODOS}

Se han revisado los 78 tumores testiculares diagnosticados en nuestro hospital entre febrero de 1993 y diciembre del 2005, analizando los 66 casos (84.6\%) que fueron de estirpe germinal.

Según el censo 2001 realizado por el INE, la población masculina de la provincia era de 323.541 varones. En nuestro estudio se ha considerado como población de riesgo para los TGT los varones con edades comprendida entre 15 y 69 años, por lo que la población masculina dependiente de nuestro Centro, desestimadas las edades extremas, se reduce a 83.460 varones (3). La Sanidad Pública en la provincia de Badajoz se divide en cuatro Áreas de Salud, el 82\% de los pacientes de nuestra serie, procedían del Área de Badajoz.

En estos 66 pacientes hemos registrado; antecedentes personales y familiares, ecografía testicular, analítica con marcadores séricos pre y postorquiectomía y estudio de extensión mediante Rx de tórax y TAC abdomino-pélvico. En algunos casos, la Rx de tórax se completó con TAC torácico, especialmente en los casos con elevación de marcadores. En los pacientes con valores de $\beta$-HCG superiores a 10.000, se realizó RMN de cráneo (2).

El tipo histológico tumoral se determinó por la Clasificación de la OMS y el estadiaje tumoral se estableció por el Sistema TNM del 1997 (4). Como clasificación pronóstica se ha utilizado la del IGCCCG de 1997 (5), tomando las decisiones terapéuticas en base a dicha clasificación.

El procesamiento estadístico se ha efectuado con el programa SPSS v. 12 sobre la base de datos de pacientes creada para este trabajo. 


\section{RESULTADOS}

De los 66 casos estudiados, 54 pacientes pertenecían a nuestra Área de Salud (82\%) acumulándose la mayor parte de los casos en los núcleos de mayor población (79.6\%). Otro dato significativo es el incremento del número de casos diagnosticados en los últimos años del estudio (Figura 1).

Sobre los factores de riesgo, se pudo recoger la información en 52 pacientes. En 45 de ellos (86.5\%) no existían factores relacionados. En 3 ocasiones $(5.7 \%)$ se encontró historia de criptorquidia, en 2 enfermos $(3.8 \%)$ antecedentes familiares y en otros 2 casos (3.8\%) infertilidad.

El síntoma mas frecuente fue el hallazgo de una masa testicular $(47.3 \%)$, seguido de dolor testicular (19.3\%) y masa testicular-dolor (15.7\%). El 5.2\% se diagnosticó por la clínica metastásica. Un 12.2\% ya diagnosticado, fue derivado desde otro Centro para tratamiento.

Se realizaron 64 orquiectomías radicales programadas vía inguinal, una orquiectomía diferida tras quimioterapia y una orquiectomia escrotal de urgencia por traumatismo.

Respecto a la lateralidad tumoral, fue sensiblemente mas frecuente la afectación del testículo izquierdo con 34 casos (51.5\%); 30 casos correspondieron al teste derecho $(45.5 \%)$; un caso con afectación sincrónica bilateral y en dos pacientes $(3 \%)$, hubo progresión de la enfermedad con afectación bilateral.

La edad media de los pacientes fue de 32 años (16-80 años). En los tumores germinales no se- minomatosos, la edad media al diagnóstico fue mas precoz, 30 años (16-78 años), en comparación a los 33 años de media (19-80 años) en los tumores seminomatosos. En los mayores de 55 años el seminoma fue el tipo de TGT más diagnosticado (Figuras 2 y 3).

El tipo histológico más frecuente lo constituyen los tumores germinales no seminomatosos $(64.8 \%)$ frente al $45.3 \%$ de los TGS. Dentro de los TGNS, se pudo diferenciar el porcentaje de las formas puras $(57.1 \%)$ que predominaron sobre las mixtas $(42.8 \%)$. Individualmente, el seminoma y el carcinoma embrionario fueron los dos tipos de tumores más diagnosticados. En la Tabla I se muestran en detalle, los diagnósticos anatomopatológicos de las orquiectomías realizadas.

Se pudo recoger la información acerca del estadio en 57 pacientes, siendo el estadio I el más diagnosticado entre los tumores germinales seminomatosos (72\%), como en los no seminomatosos $(68.7 \%)$. Se objetivó un incremento de los casos en estadio I en los últimos años del estudio. Los pacientes diagnosticados en fase avanzada de la enfermedad fue de un $28 \%$ en los TGS frente al $31.2 \%$ de los TGNS, en los que el estadio II se dió en 9 pacientes $116 \%$ de seminomas versus 15.6 de los no seminomas) y 8 pacientes debutaron en estadio III (12\% versus $15 \%$ ) (Figuras 4 y 5 ).

Un $92 \%$ recibió tratamiento adyuvante con finalidad curativa/preventiva, de acuerdo al protocolo del Grupo Español de Tumores Germinales. En el $8 \%$ restante de pacientes, únicamente se practicó la orquiectomía radical, pasando a política de seguimiento.

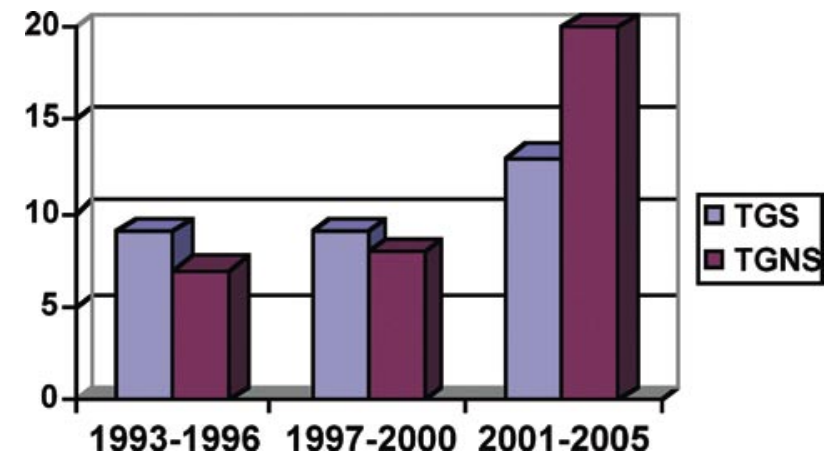

FIGURA 1. Incidencia tumoral. En los últimos años, se observa un aumento en el número de casos, sobre todo a expensa de los tumores germinales no seminomatosos.

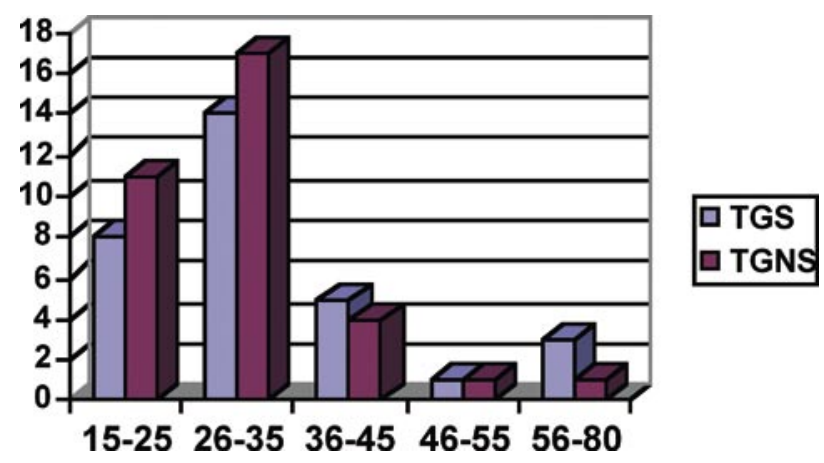

FIGURA 2. Edad del paciente y tipo tumoral. 


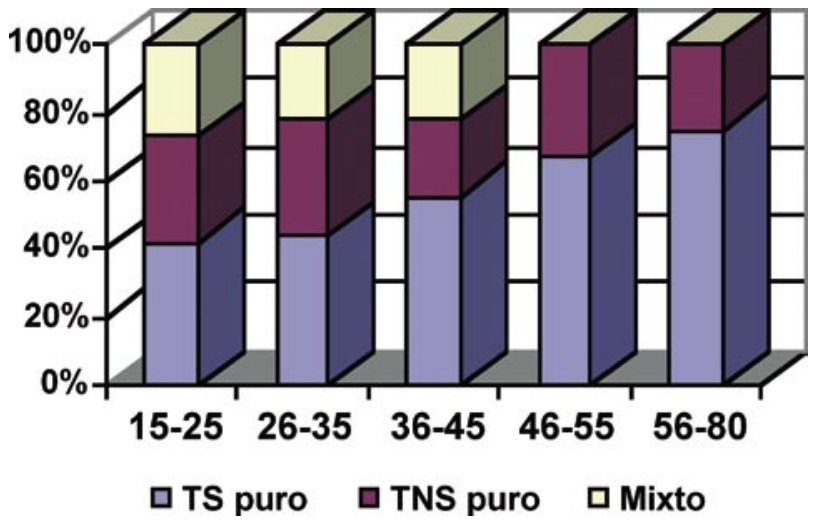

FIGURA 3. Relación entre el tipo histológico lformas puras y mixtas) y la edad al diagnóstico.

Los seminomas en estadio I fueron tratados con radioterapia en el $22.2 \%$ de los casos, la mayoría de ellos en los primeros años del estudio. Se aplicó quimioterapia adyuvante en el $55.5 \%$ y pasaron a política de seguimiento el $16.6 \%$ frente al $13.6 \%$ de los no seminomatosos en estadio I. El resto de los pacientes, recibió quimioterapia con fines curativos.

En 5 casos fue necesario practicar resección de masas residuales tras QT, con los siguientes resultados: un paciente con necrosis tumoral; 2 enfermos con teratoma (tumores primarios, seminoma y mixto) y dos paciente con tumor germinal mixto (diagnóstico primario, TGNS).

El seguimiento del paciente debe ser intensivo en los primeros años, cuando hay mayor riesgo de recurrencia de la enfermedad. Posteriormente, debido a la existencia de un periodo prolongado de la-

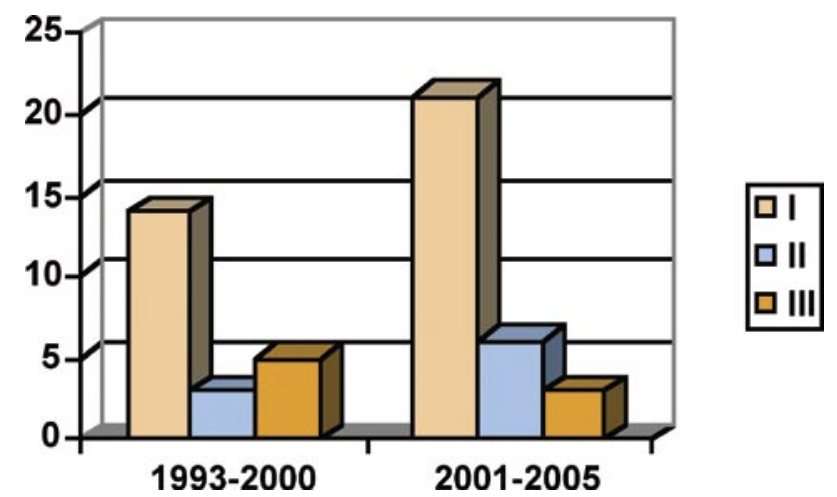

FIGURA 5. Evolución de los estadios tumorales al diagnostico durante el estudio.

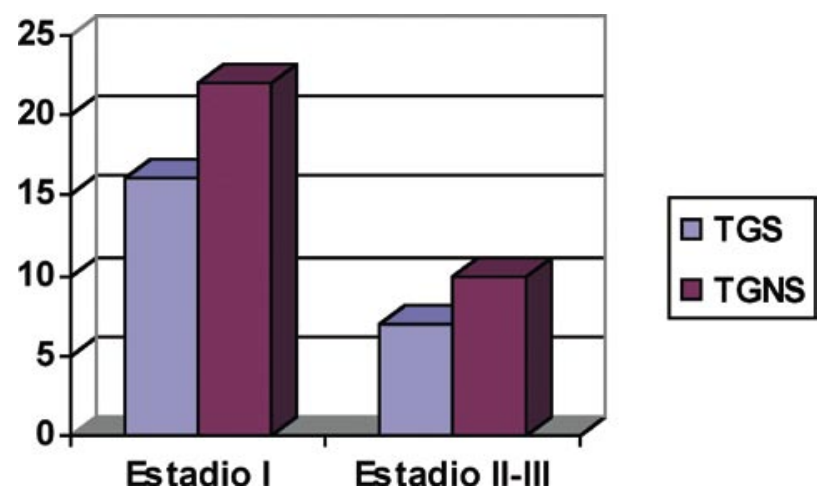

FIGURA 4. Relación entre el estadio y tipo tumoral.

tencia para la aparición de metástasis y/o segundos tumores (inducidos por el tratamiento y del testículo contralateral), se recomienda una vigilancia a largo plazo (4). En nuestra serie se ha cumplido la política de seguimiento del Grupo Español de Tumores Germinales.

La supervivencia se ha evaluado en 58 casos $(87,8 \%)$, encontrando 4 éxitus; 1 caso de muerte por progresión de la enfermedad y 3 casos de fallecimiento por enfermedades interrecurrentes: un paciente en el postoperatorio de una estenosis aórtica, otro caso por hemorragia digestiva alta en el contexto de una hepatopatía alcohólica y el último caso por accidente cerebro-vascular (ACV). Los restantes 54 casos $(93,1 \%)$ están libre de enfermedad, de los cuales, el $25 \%$ llevan mas de 5 años desde el diagnóstico.

\section{DISCUSIÓN}

Es bien conocido el importante problema de salud pública que supone el cáncer, tanto por la mortalidad que produce como por la morbilidad y años productivos de vida perdidos (APVP).

Para poder llevar a cabo acciones de prevención y control de forma más efectiva sobre esta patología es necesario obtener información de calidad. Esta información permitirá realizar estudios epidemiológicos para saber cuál o cuáles son las causas que determinan la presentación de un tipo de tumor, poniendo en marcha planes de diagnóstico precoz y planificación de las estrategias sanitarias.

Hay estudios que relacionan la criptorquidia (8) y ciertos factores prenatales y perinatales (9), peso al nacer, edad de la madre, si es o no fumadora, con un mayor riesgo de padecer cáncer testicular. 
En nuestra serie había dos casos $(3.8 \%)$ con antecedentes de criptorquidia unilateral y un caso bilateral, intervenidos en la infancia. Con una anatomía patológica de seminoma en dos pacientes y un tumor germinal mixto.

También se considera un factor de riesgo la existencia de cáncer testicular entre familiares de primer grado, más aún entre hermanos, donde pudiese existir algún otro factor involucrado (11). En nuestra serie, se registraron dos casos $(3.8 \%)$ de TGNS en dos hermanos, ambos diagnosticados en la tercera década, con un bajo estadio tumoral y con un intervalo de un año entre ambos diagnósticos.

Dos casos de TGT (3.8\%) fueron diagnosticados en el contexto de un estudio por infertilidad, con una histología tumoral de seminoma y TGNS. En un estudio americano (10), analizaron la incidencia tumoral en pacientes con seminograma anormal e infertilidad, encontrando un aumento en la incidencia tumoral, cuando lo comparaban con la población general, pudiendo ser el cáncer una de las causas de la infertilidad.
Distintos estudios $(12,13,15)$ informan una incidencia de cáncer testicular bilateral de 1,5-5\%, con un intervalo medio entre ambos diagnósticos de 50-70 meses; los tipos histológicos mas frecuentes en el segundo tumor fueron el seminoma y el teratoma. El tratamiento inicial no parece afectar a la terapéutica aplicada en el segundo tumor que suele presentar un estadio bajo, obteniendo muy buenos resultados y una supervivencia elevada. En nuestra serie se dieron tres casos $(5.7 \%)$, de los cuales un paciente presentó un TGT bilateral síncrono, con histología de TGNS. Los otros dos casos tuvieron afectación metacrónica del segundo testículo, con un periodo de latencia entre ambos diagnósticos de dos y tres años. La histología fue de teratoma inmaduro (ler diagnostico) y seminoma $\left(2^{\circ}\right.$ diagnóstico) en un paciente, y dos seminomas en el otro enfermo.

Aproximadamente el $5 \%$ de los pacientes con TGT presentan cáncer no invasivo (cancer in situ y neoplasia intraepitelial -TIN-) en el testículo contralateral, de los cuales alrededor del 50\% desarrollan un TGT a los cinco años. En Dinamarca (14),

TABLA I. TIPO HISTOLÓGICO DE LOS TUMORES GERMINALES TESTICULARES.

\begin{tabular}{|l|c|c|}
\hline TIPO HISTOLÓGICO & N $^{\circ}$ Pacientes & Porcentaje \\
\hline Seminomas puros & $\mathbf{2 9}$ & $\mathbf{4 3 . 9}$ \\
\hline Seminomas clásico & 28 & 42.4 \\
\hline Seminomas anaplásico & 1 & 1.5 \\
\hline Otras estirpes germinales puras & $\mathbf{2 0}$ & $\mathbf{3 0 . 3}$ \\
\hline Teratoma maduro & 3 & 4.5 \\
\hline Teratoma inmaduro & 2 & 3 \\
\hline Carcinoma embrionario & 10 & 15.5 \\
\hline Coriocarcinoma & 2 & 3 \\
\hline Tumor del seno endodérmico & 3 & 4.5 \\
\hline Tumores mixtos & 15 & $\mathbf{2 2 . 7}$ \\
\hline Seminoma + T seno endodérmico & 1 & 1.5 \\
\hline Seminoma + Teratoma & 2 & 3 \\
\hline Seminoma + Ca embrionario & 4 & 6 \\
\hline Ca embrionario + Teratoma & 4 & 6 \\
\hline Ca embrionario + T seno endodérmico & 1 & 1.5 \\
\hline T seno endodérmico + Teratoma & 1 & 1.5 \\
\hline Valores perdidos & $\mathbf{2}$ & $\mathbf{3}$ \\
\hline
\end{tabular}


se efectúa de forma sistemática biopsia del testículo contralateral durante la orquiectomía; si la biopsia es positiva de TIN realizan un tratamiento especificó con radioterapia localizada a bajas dosis (18-20 Gy), aportando buenos resultados y pocos efectos secundarios. Se evita así la realización de otra orquiectomía, (con el consiguiente déficit hormonal y efecto emocional) y se reduce la toxicidad derivada de nuevos tratamientos. Sin embargo, Hentrich (12) no recomienda realizar biopsia del testículo contralateral de forma rutinaria, argumentando que un segundo diagnóstico de TGT presenta un pronóstico excelente y que, independientemente de la existencia de $T I N$, es necesario realizar controles estrictos, además del impacto emocional que podría provocar en el paciente, el conocimiento de un TIN contralateral, y la afectación de la fertilidad por la irradiación local a la que se sometería. En nuestra serie, sólo se realizaron dos biopsias testiculares contralaterales durante la orquiectomía, ambas por la existencia de microcalcificaciones, siendo negativas de TIN.

La media de edad al diagnóstico fue más precoz en los no seminomatosos, 30 años con respecto a los 33 años de los seminomas, al igual que en otras series $(6,7,18)$. Si diferenciamos entre los tumores mixtos y puros, las formas mixtas son más frecuentes en los pacientes mas jóvenes, destacando que entre los mayores de 55 años sólo fueron diagnosticados seminomas.

Al contrario de lo que ocurre en otras series españolas, en nuestro estudio es sensiblemente más frecuente la afectación tumoral en el testículo izquierdo $(6,7)$. Los tumores germinales testiculares no seminomatosos, fueron el tipo histológico más frecuente $(64.8 \%)$, similar a lo que se observa en otros autores $(6,7,8)$. La prevalencia del tipo tumoral, parece estar más influida por ciertos factores de riesgo, como la criptorquidia y la edad maternal avanzada en los $\operatorname{TGTS}(2,9)$.

Como viene reflejado en varios estudios $(7,17,21)$ europeos realizados en la última década, se observa un incremento en la incidencia de las neoplasias germinales de testículo. Algunos autores apuntan al aumento de todos los tipos de tumores (18), mientras que German-Lluch (6) objetiva un ligero aumento de los TGNS sobre los seminomas.

En nuestra serie, en los últimos cuatro años se diagnosticaron el $50 \%$ de los TGT, con predominio de los no seminomatosos, una alta incidencia para la población masculina de nuestra Área, si consideramos que la incidencia en nuestro país estaría entre 1,5-2 casos por 100.000 habitantes/año. En este sentido, hay que destacar la incidencia especialmente elevada en una zona concreta del Área de Salud, con una población de riesgo de 2.000 individuos, donde se han diagnosticado 4 casos en este periodo de cuatro años.

El diagnóstico precoz observado en los diferentes estudios, confiere a la enfermedad un pronóstico aún mejor, incluso en los grupos mas desfavorecidos (IGCCCG). German-Lluch (6) encuentra una relación significativa entre el intervalo del primer síntoma /orquiectomía y el pronóstico de la enfermedad.

El estadio I fue la forma de presentación del tumor más frecuente durante nuestro estudio, sobre todo en los últimos 5 años $(63.6 \%)$ con respecto al $53.8 \%$ durante la década de los 90 , como se describe en otros estudios (21). Los seminomas fueros diagnosticados en un estadio más inicial $(72 \%)$, mientras que los no seminomatosos se presentaron como enfermedad avanzada en el $31.2 \%$ de los pacientes. Molina (17) presenta en su serie esta tendencia tumoral, con disminución de estadios avanzados y recurrencia de la enfermedad, favorecido por el uso incrementado de la quimioterapia adyuvante.

El tratamiento oncológico fue aplicado en función al pronóstico tumoral; seleccionando los casos, en el $27.8 \%$ de los estadio I de seminoma y teratoma maduro, sólo se realizó orquiectomía sin constatar en estos casos (sin factores de riesgo), progresión de la enfermedad, hasta la finalización del estudio.

La quimioterapia adyuvante con carboplatino en los estadios I del seminoma con factores de peor pronóstico puede ser aplicada para la prevención de progresión (6) Aparicio (22) y cols. la aplican al estadio I del seminoma con factores de riesgo, siendo efectiva en la reducción de tasa de recidiva tumoral, con buena tolerancia y bajo grado de toxicidad.

Como ya se ha comentado, debido a la existencia de un periodo prolongado de latencia para la aparición de metástasis y/o tumor testicular contralateral, se recomienda una vigilancia a largo plazo. El diagnóstico de recidiva tumoral o de masa residual activa postratamiento se ha beneficiado con la utilización de PET-TC (20). De los casos con peor pronóstico que se presentaron en nuestra serie $(15.1 \%)$, sólo se ha registrado un caso de muerte por progresión, similar a la comunicada por otros autores (6-8).

\section{CONCLUSIONES}

El incremento de incidencia objetivado en los últimos años obliga a intensificar los estudios que permitan determinar cuales son los factores que lo favorecen. 
En el presente estudio se verifica un diagnóstico más precoz de la enfermedad, con mayor número de casos en estadios iniciales que confieren un mejor pronóstico.

El abordaje multidisciplinar y los protocolos de tratamiento oncológico aplicados, con menor riesgo y toxicidad, proporcionan una elevada tasa de éxito terapéutico, por lo que se puede considerar que los tumores testiculares germinales constituyen un modelo de cáncer curable, incluso en enfermedad metastásica.

\section{BIBLIOGRAFIAA y LECTURAS RECOMENDADAS ( ${ }^{*}$ lectura de interés $y^{* *}$ lectura fundamental)}

1. RICHIE, J.P.; STEELE, G.S.: "Campbell Urología”. 8 ediccion. Pag 3147-3179. Ed. Medica Panamericana. Buenos Aires, 2005.

2. RESEL ESTÉVEZ, L.; MORENO SIERRA, J. y cols.: "Tratado de Oncologia Urológica". Pag 935-996. $1^{\text {a }}$ ed. Sanidad y ediciones, S.L. Madrid, 2003.

3. INE. Instituto nacional de Estadística. Censo Municipal 2001. En: http://www.ine.es

4. LAGUNA, M.P.; KLEPP, O.; HORWICH, A. y cols.: "Guideline on testicular cancer". European Association of urology, 2004.

5. INTERNACIONAL GERM CELL CANCER COLLABORATIVE GROUP.: "Internacional Germ Cell consensus Classification a prognostic factor-based staging systen for metastasic germ cell cancer". J. Clin. Oncol., 15: 594, 1997.

**6. GERMAN-LLUCH, J.R.; GARCIA DEL MURO, X.; MARATO, P. y cols.: "Spanish Germ-Cell Cancer Group. Clinical pattern and therapeutic results achieved in 1490 patients with germ cell tumours of testis: the experience of the Spanish Germ-Cell Cancer Group”. Eur. Urol., 42: 553, 2002.

7. DIZ RODRÍGUEZ, R.; VIRSEDACHAMORRO, M.; PAÑOS LOZANO, P. y cols.: "Tumores testiculares. Evaluación de la experiencia durante 25 años en un hospital militar". Actas Urol. Esp., 29: 457, 2005.

8. FERNÁNDEZ GOMEZ, J.M.; GUATE ORTIZ, J.L.; MARTÍN HUESCAR, A. y cols.: "Presentación clínica del cáncer germinal de testículo". Arch. Esp. Urol., 55: 915, 2002.

9. MOLLER, H.; SKAKKEBAEK, N.E.: "Testicular cancer and cryptorchidism in relation to prenatal factors: case-control studies in Denmark". Cancer Causes Control, 8: 904, 1997.
10. ENGHISH, P.B.; GOLDBERT, D.E.; WOLFF, C.: "Prenatal and birth characteristics in relation to testicular cancer risk among males born between 1960 and 1995 in California". Cancer Causes Control, 14: 815, 2003.

11. REMAN, J.D.; NOBERT, C.F.; GOLDSTEIN, M.: "Increased incidence of testicular cancer in men presenting whit infertility and abnormal semen analysis". J. Urol., 174: 1819, 2005.

12. WESTERGAARD, T.; OLSEN, J.H.; FRISCH, M. y cols.: "Cancer risk in father and brother of testicular cancer patients in Dermark. A population-based study". Int. J. Cancer, 66: 627, 1996.

13. HENTRICH, M.; WEBER, N.; BERGSDORF, T. y cols.: "Management and outcome of bilateral testicular germ cell tumors: Twenty-five year experience in Munich". Acta Oncol., 44: 529, 2005.

*14. PAMENTER, B.; DE BONO, J.S.; BROWN, M. y cols.: "Bilateral testicular cancer: a preventable problem?. Experience from a large cancer centre". Br. J. Urol., 92: 43, 2003.

*15. VON DER MASSE, H.: "Is a contralateral testicular biopsy in patients with unilateral germ cell testicular cancer indicated as a routine procedure?". Act. Oncol., 44: 523, 2005.

16. HOLZBEIERLEIN, J.M.; SOGANI, P.C.; SHEINFELD, J.: "Histology and clinical outcomes in patients with bilateral testicular germ cell tumors: the Memorial Sloan Kettering cancer Center experience 1950-2001”. J. Urol., 169: 2122, 2003.

**17. MOLINA SAERA, J.; APARICIO URTASUN, J.; DIAZ BEVERIDGE, R. y cols.: "Epidemiological pattern and time trends in testicular germ-cell tumors: a single institution 20-year experience". Clin. Transl. Oncol., 8: 588, 2006.

18. HERNES, E.H.; HARSTAD, K.; FOSSA.: "Changing incidence and delay of testicular cancer in southern Norway (1981-1992)”. Eur. Urol., 30: 349, 1996.

19. FERNANDEZ GOMEZ, J.M.; ESCALF BRAMADA, S.; GUATE ORTIZ, J.L. y cols.: "Tratamiento urológico del cáncer germinal testicular". Arch. Esp. Urol., 55: 927, 2002.

20. SANCHEZ ZUDAIRE, J.J.; FERNANDEZ, J.M. y cols.: "F-fluoro-2-desoxiglucose-positron emisión tomography in the evaluation of non seminomatous germ cell tumours at relapse". Br. J. Urol., 89: 912, 2002.

**21. HORWICH, A.; SHIPLEY, J.; HUDDART, R.: "Testicular germ-cell cancer". Lancet, 367: 754, 2006.

22. APARICIO, J.; GERMA, J.R.; GARCÍA DEL MURO, X. y cols.: "Risk- adapted management for patients with clinical stage seminoma: the second spanish germ cell cancer cooperative group study”. J. Clin. Oncol., 23: 8717, 2005. 